\title{
A case of ovarian hyperstimulation syndrome with spontaneous hemoperitoneum
}

\author{
Prasad R. Lele ${ }^{1 *}$, Manoj Kumar Tangri²
}

\begin{abstract}
${ }^{1}$ Senior Advisor (Obs and Gyn), Reproductive Medicine Specialist, Command Hospital, Associate Professor, King Gorge Medical University, Lucknow, Uttar Pradesh, India

${ }^{2}$ Senior Advisor (Obs and Gyn), Gynaec Oncosurgeon, Command Hospital , Associate Professor, West Bengal Medical University, Kolkata, Bengal, India
\end{abstract}

Received: 04 December 2016

Accepted: 28 December 2016

\author{
*Correspondence: \\ Dr. Prasad R. Lele, \\ E-mail: prasadlele@hotmail.com
}

Copyright: (C) the author(s), publisher and licensee Medip Academy. This is an open-access article distributed under the terms of the Creative Commons Attribution Non-Commercial License, which permits unrestricted non-commercial use, distribution, and reproduction in any medium, provided the original work is properly cited.

\begin{abstract}
We report a case of complicated severe OHSS that developed spontaneous hemoperitoneum. This was a potentially fatal complication of pharmacological stimulation of ovary in assisted reproduction. Here the life was saved, oophorectomy was prevented and fertility preserved by timely diagnosis and conservative surgery.
\end{abstract}

Keywords: Haemoperitoneum, OHSS

\section{INTRODUCTION}

Ovarian hyperstimulation syndrome (OHSS) is a potentially life-threatening complication of pharmacological ovarian stimulation. ${ }^{1}$ The easy availability of assisted reproductive technology (ART) with success of infertility treatment has resulted in an increase in ovulation induction and its complications including OHSS.

The OHSS has been classified in three different categories such as mild, moderate and severe. In $33 \%$ of IVF cycles have been reported to be associated with mild forms of OHSS, whereas the more severe forms have been reported in $3.1 \%$ to $8.0 \%$ of IVF cycles. ${ }^{2}$ Severe forms complicate $\sim 1 \%$ of IVF cycles and are characterized by a massive ovarian enlargement together with a fluid shift into extravascular compartments responsible for the development of ascites, sometimes pleural and/or pericardial effusion, hypovolemia, oliguria, and hydroelectrolytic disorders. ${ }^{3}$

These hyperstimulated ovaries may rarely rupture or undergo torsion, which often misdiagnosed and reported to range from $0.08 \%$ to $0.2 \%{ }^{4}$ Surgical intervention maintaining ovarian integrity and reserve is challenging because of the friability of the tissue. We present a case with conservative surgical management of spontaneous hemoperitoneum following rupture of ovarian follicular vessel in a patient with OHSS.

\section{CASE REPORT}

A 28-year-old woman a case of anovulatory secondary infertility underwent ovulation induction with gonadotropins and regular ultrasound guided follicular monitoring from D2 of her menstrual cycle for intrauterine insemination (IUI). On D12 of her menstrual cycle when she had three follicles of 18 to $21 \mathrm{~mm}$ size with endometrium of $9 \mathrm{~mm}$ she was then given Inj HCG (10000 IU) as surrogate for ovulation and her IUI was done after 36 hrs. Patient presented 10 days after IUI with nausea, mild pain and distension of abdomen. Ultrasound revealed bilateral ovarian enlargement (right ovary $6.8 \mathrm{x}$ $7.2 \mathrm{cms}$ and left ovary $7.2 \times 8.3 \mathrm{cms}$ ) with anechoic peritoneal collection predominantly to pouch of Douglas. Her hemoglobin and PCV were $15 \mathrm{gm} / \mathrm{dl}$ and $45 \%$ respectively. She was diagnosed as a case of moderate 
OHSS, admitted and treated conservatively with plasma expanders (Human Albumin 20\%) and anti-emetics and $\mathrm{H} 2$ blockers according to our department guidelines.

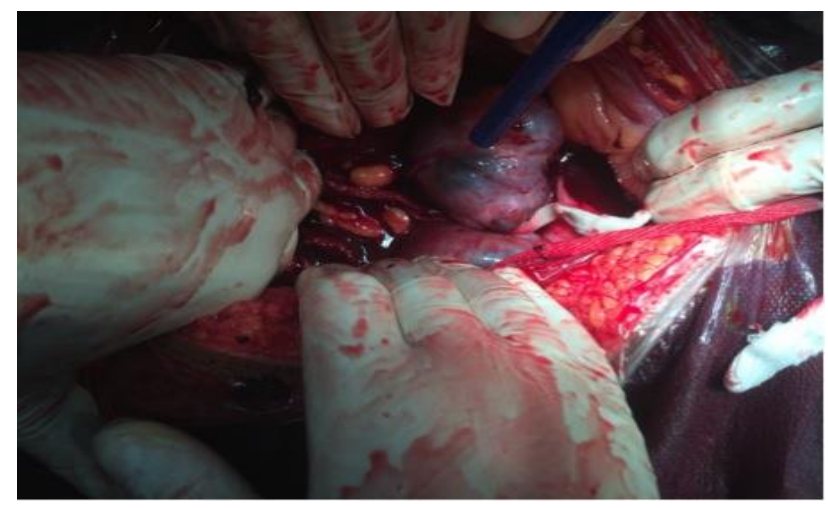

Figure 1: At exploratory laparotomy, haemoperitoneum with bilaterally enlarged ovaries.

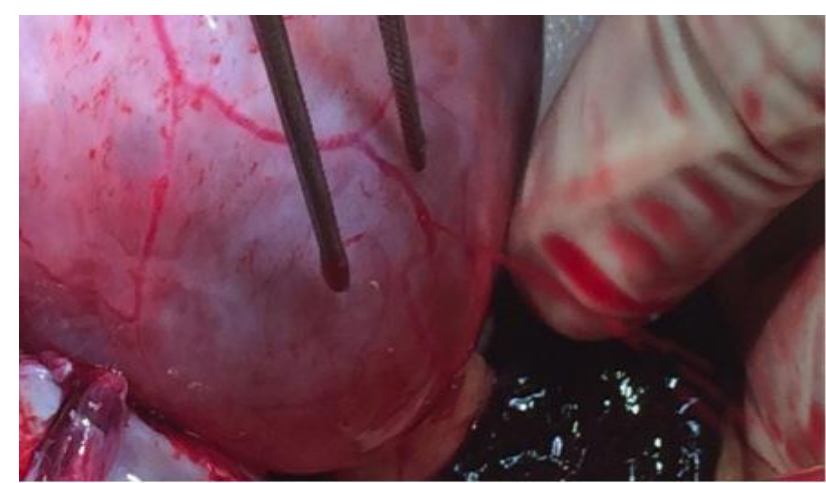

Figure 2: Enlarged right ovary with bleeder (Spurter bleed).

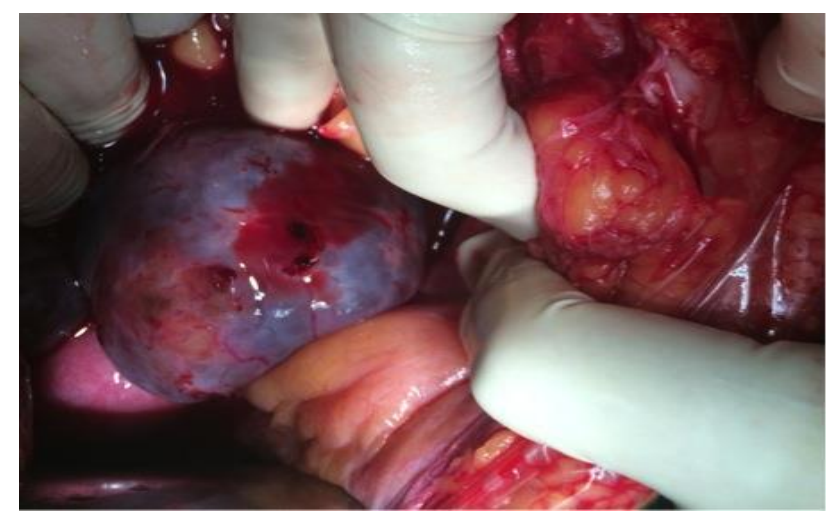

Figure 3: Enlarged left ovary with tear in follicular wall and bleeding (Oozing bleed).

The patient was warned about the complications and asked restrain her movements in the ward. Daily her temperature, pulse, blood pressure charting haematocrit, renal function tests along with bedside trans abdominal sonography for severity of ascites were checked and were within limits. But her condition deteriorated 4 days later, as she suddenly developed syncope with severe lower abdominal pain with distension. Her hemoglobin dropped to $5.8 \mathrm{gm} / \mathrm{dl}$. An ultrasound done immediately showed enlarged ovaries right ovary $8.8 \times 9.2 \mathrm{cms}$ and left ovary $9.2 \times 8.3 \mathrm{cms}$ with excessive fluid in the abdomen and pelvic area with internal echoes consistent with the appearance of blood clots. A diagnosis of hemoperitoneum was made and confirmed with USG guided bedside sanguineous ascetic tap.

Emergency exploratory laparotomy was performed with midline infraumbilical vertical incision. Intra-op approximately $1700 \mathrm{ml}$ of blood-stained fluid and multiple clots were present in cul-de-sac (Figure 1). Both ovaries were enlarged with multiple follicles. The right ovary was $10 \times 9 \mathrm{cms}$ with point bleeder (Figure 2) while the left ovary was $9 \times 8 \mathrm{cms}$ and there was active bleeding from an irregular tear on follicular wall (Figure $3)$. Right ovarian follicular bleeder was cauterized and then sutured with 3/0 Polyglactin 910 suture and the left ovary was also managed with similar approach. After ensuring hemostasis, abdomen was closed with peritoneal drain in situ. Transfusion therapy was given and her hemoglobin increased to $9.1 \mathrm{gm} / \mathrm{dl}$. She made uneventful post-op recovery and discharged on hematinics. Ultrasound done on $8^{\text {th }}$ post op day revealed minimal bilateral ovarian enlargement with scanty fluid in cul-desac.

\section{DISCUSSION}

OHSS is the most serious complication of controlled ovarian stimulation (COS) as part of assisted reproductive technologies (ART). While the safety and efficacy of ART is well established, physicians should always be aware of the risk in severe form of OHSS, complicated further by ovarian accident, in patients undergoing COS, as it can be fatal. It usually presents after HCG administration in ovulation induction programs and early in the first trimester if conception occurs. ${ }^{5}$ Ovarian accidents like torsion or rupture are rare but recognized serious complication of OHSS. In a young otherwise healthy infertile patient they pose a challenge to conserve the ovary and preserve fertility. ${ }^{6}$

Ovarian rupture complicating OHSS is rare it may result from the rapidly enlarging vascular ovary with friable edematous stromal and cortical tissue. ${ }^{4}$ Active bleeding arises from fissures between intact follicles and from deeper stromal vessels, or from enlarging follicular capsule as in our patient. ${ }^{7}$ Diagnostic dilemma may be encountered because ruptured ectopic pregnancy or luteal cyst can also cause intraperitoneal hemorrhage. ${ }^{8}$

In a series by Hertzberg et al of 9 patients with hemoperitoneum who were ultimately diagnosed with ruptured hemorrhagic ovarian cyst, the diagnosis was prospectively made on sonography or computed tomography in only 4 patients $(44 \%) .{ }^{9}$ Diagnosis of hemoperitoneum can be done by USG and differentiated from ascites by the presence of echogenic shadows 
representing blood clots; however, sometimes hemoperitoneum may be anechoic, particularly in the very early or late stages and thus may mimic ascites, ${ }^{8}$ In this case, clinical judgment plays a major role in early diagnosis and intervention. The aim of conservative surgical management of the ovaries in these cases is mainly to control the bleeding and preserve ovarian tissue. Hemostasis with sutures or electrocautery may not be easy owing to the soft friable nature of the enlarged oedematous ovary. ${ }^{4}$ In our case, the ovarian follicular wall had ruptured bilaterally resulting into extensive internal bleeding. Timely diagnosis, decision for exploratory laparotomy with liberal midline incision, extreme care while handling the friable enlarged ovaries and the meticulous suturing to achieve hemostasis resulted in bilateral ovarian preservation.

For OHSS free clinics $\mathrm{COH}$ regimens should be highly individualized, carefully monitored. Preventive measures should be tailored and instituted in the high-risk cases. Use of dopamine agonist, IV calcium gluconate, IV human albumin are pharmaceutical tools in the armamentarium of a clinician for prevention, combined with in-vitro oocyte maturation and oocyte, embryo cryopreservation by vitrification and elective single embryo transfer, most of the serious complications inflicted by ART can be averted. ${ }^{10}$ In summary, we presented a case of complicated severe OHSS with spontaneous hemoperitoneum. Ovarian salvage could be achieved by timely diagnosis and conservative surgery. The cause of rupture is still enigmatic and emphasis should be there careful handling of patient with these enlarged friable ovaries to avoid catastrophe.

Funding: No funding sources

Conflict of interest: None declared

Ethical approval: Not required

\section{REFERENCES}

1. Devroey P, Adriaensen P. OHSS Free Clinic Facts Views Vis Obgyn. 2011;3(1):43-5.
2. Humaidan P, Quatartarolo J, Papanikolaou EG. Preventing ovarian hyperstimulation syndrome: guidance for the clinician. Fertil Steril. 2010;94(2):389-400.

3. Papanikolaou EG, Pozzobon C, Kolibianakis EM, et al. Incidence and prediction of ovarian hyperstimulation syndrome in women undergoing gonadotropin-releasing hormone antagonist in vitro fertilization cycles. Fertil Steril. 2006;85:112-20.

4. Al Omari W, Ghazal-Aswad S, Sidky IH, Al Bassam MK. Ovarian salvage in bilaterally complicated severe ovarian hyperstimulation syndrome. Fertil Steril. 2011;96(2):e77-9.

5. Fiedler K, Ezcurra D. Predicting and preventing ovarian hyperstimulation syndrome (OHSS): the need for individualized not standardized treatment. Reprod Biol Endocrinol. 2012;10:32.

6. Krishnan S, Kaur H, Bali J, Rao K. Ovarian torsion in infertility management - Missing the diagnosis means losing the ovary: A high price to pay $\mathrm{J}$ Hum Reprod Sci. 2011;4(1):39-42.

7. Maxwell, K.N., Cholst, I.N., Rosenwaks, Z.The incidence of both serious and minor complications in young women undergoing oocyte donation. Fertil Steril. 2008;90:2165-71.

8. Gunabushanam G, Mandal K, Lal S. Hemoperitoneum from ruptured cyst in a hyperstimulated ovary: a sonographic mimic of ovarian hyperstimulation syndrome. J Clin Ultrasound. 2007;35(5):281-3.

9. Hertzberg, B.S., Kliewer, M.A., and Paulson, E.K. Ovarian cysts rupture causing hemoperitoneum: imaging features and the potential for misdiagnosis. Abdom Imaging. 1999;24:304-8.

10. Naredi N, Talwar P, Sandeep K. VEGF antagonist for the prevention of ovarian hyperstimulation syndrome: Current status. Med J Armed Forces India. 2014;70(1):58-63.

Cite this article as: Lele PR, Tangri MK. A case of ovarian hyperstimulation syndrome with spontaneous hemoperitoneum. Int J Reprod Contracept Obstet Gynecol 2017;6:766-8. 